International Journal of Modern Physics A

(C) World Scientific Publishing Company

\title{
Search for rare leptonic B decays at the Tevatron
}

\author{
Ralf Bernhard
}

Physics Institute of the University of Zürich, Winterthurerstr. 190

CH-8057 Zürich, Switzerland

\begin{abstract}
Results of a search for the Flavor-Changing Neutral Current decay $B_{s, d}^{0} \rightarrow \mu^{+} \mu^{-}$using $p \bar{p}$ collision data at $\sqrt{s}=1.96 \mathrm{TeV}$ collected at Fermilab Tevatron collider by the $\mathrm{CDF}$ and $\mathrm{D} \varnothing$ detectors are presented. CDF reports upper limits on $\mathcal{B}\left(B_{s}^{0} \rightarrow \mu^{+} \mu^{-}\right) \leq$ $7.5 \cdot 10^{-7}$ and $\mathcal{B}\left(B_{d}^{0} \rightarrow \mu^{+} \mu^{-}\right) \leq 1.9 \cdot 10^{-7}$ at the $95 \%$ C.L. using $171 \mathrm{pb}^{-1}$. The D $\varnothing$ Collaboration used $240 \mathrm{pb}^{-1}$ to set an even more stringent limit on the branching ratio for $B_{s}^{0} \rightarrow \mu^{+} \mu^{-}$of $5.0 \cdot 10^{-7}$ at the $95 \%$ C.L..
\end{abstract}

Keywords: Tevatron; B physics; rare decays.

\section{Introduction}

The Tevatron Run II at Fermilab started to deliver $p \bar{p}$ collisions at $\sqrt{s}=1.96 \mathrm{TeV}$ in April 2002. The $b \bar{b}$ production cross section at this center of mass energy is very large $(\approx 150 \mu \mathrm{b})$ when compared to the typical $e^{+} e^{-}$cross sections at the $\Upsilon(4 S)(\approx$ $1 \mathrm{nb})$ and $Z^{0}(\approx 7 \mathrm{nb})$ resonances. The $b \bar{b}$ production rate at the current record luminosity at the Tevatron of $\approx 10^{32} \mathrm{~cm}^{-1} \mathrm{sec}^{-1}$ is $10 \mathrm{kHz}$. At hadron colliders there is also the advantage of producing all b-flavored species from the light $B_{u}^{+}$and $B_{d}^{0}$ mesons to the heavier $B_{s}^{0}$ and $B_{c}^{+}$meson as well as $b$ baryons such as $\Lambda_{b}^{0}$. On the other hand, the problem of the hadronic environment is the high level of background due a large inelastic $p \bar{p}$ cross section of $\mathcal{O}(50 \mathrm{mb})$ and the track multiplicity to up to 50 tracks/events due to the fragmentation of the hard intersection products plus that due to the underlying event or pile up of multiple events.

\section{Theoretical Introduction}

The purely leptonic decay $B_{d, s}^{0} \rightarrow \mu^{+} \mu^{-}$is a Flavor-Changing Neutral Current (FCNC) process 1 . In the Standard Model $(\mathrm{SM})$, this decay is forbidden at the tree level and proceeds at a very low rate through higher order diagrams. The SM leptonic branching fraction $(\mathcal{B})$ were calculated including QCD corrections 2 . The latest SM predictions 3 is, $\mathcal{B}\left(B_{s}^{0} \rightarrow \mu^{+} \mu^{-}\right)=(3.42 \pm 0.54) \cdot 10^{-9}$, where the error is dominated by non-perturbative hadronic uncertainties. The corresponding leptonic branching fraction for the $B_{d}^{0}$ is suppressed by an additional factor of $\left|V_{t d} / V_{t s}\right|^{2}$ leading to a SM branching ratio of $(1.00 \pm 0.14) \cdot 10^{-10}$. CDF reported in Run I the 
experimental bound for the branching fraction of $B_{s}^{0}\left(B_{d}^{0}\right)$ of $\mathcal{B}\left(B_{s}^{0}\left(B_{d}^{0}\right) \rightarrow \mu^{+} \mu^{-}\right)<$ $2.6 \cdot 10^{-6}\left(8.6 \cdot 10^{-7}\right)$ at the $95 \%$ C.L. 4 .

The decay amplitude of $B_{d, s} \rightarrow \mu^{+} \mu^{-}$can be significantly enhanced in some extensions of the SM. For instance, in the type-II two Higgs Doublet Model (2HDM), all contributions from the neutral Higgs sector cancel out and the branching fraction depends only on the charged Higgs mass $M_{H^{+}}$and $\tan \beta$ which defines the ratio of the vacuum expectation values of the Higgs field. The amplitude grows as $\tan ^{4} \beta$ [ In the Minimal Supersymmetric Standard Model (MSSM) however, $\mathcal{B}\left(B_{s}^{0} \rightarrow \mu^{+} \mu^{-}\right) \propto \tan ^{6} \beta$, leading to an enhancement of up to three orders of magnitude 67 compared to the SM, even if MSSM with minimal flavor violation (MFV) is considered, i.e., the CKM matrix is the only source of flavor violation.

\section{Event Selection}

For normalization CDF used the $b$ cross section as measured by the experiment in Run I; therefore, the preselection criteria were driven by the measurement of the cross section with $p_{T}^{B}>6 \mathrm{GeV}$ and $\left|y_{B}\right|<1.0$ for events selected by dimuon triggers. In addition a set of standard track, muon $\left(p_{T}>2 \mathrm{GeV} / c,|\eta|<0.6\right)$ and vertex quality cuts were applied. After restricting the invariant mass region of the dimuon pair to 4.669 to $5.696 \mathrm{GeV} / c^{2}, 2940$ events survive in a total integrated luminosity $\mathcal{L}$ of $171 \mathrm{pb}^{-1}$.

DØ used data collected by dimuon tiggers with two muons of opposite charge that form a common secondary 3D-vertex with an invariant mass between 4.5 and $7.0 \mathrm{GeV} / c^{2}$. Each muon candidate had to have $p_{T}>2.5 \mathrm{GeV} / c,|\eta|<2.0$ and a sufficient number of hits in the central tracking station. To ensure a similar $p_{T}$ dependence of the $\mu^{+} \mu^{-}$system in the signal and in the normalization channel, $p_{T}^{B}$ had to be greater than $5 \mathrm{GeV} / c$. After the preselection, 38,167 events survive in a integrated luminosity of $240 \mathrm{pb}^{-1}$.

\subsection{Discriminating variables}

Both experiments have chosen a set of similar discriminating variables to best exploit the properties of the decay. The long lifetime of the $B_{s}^{0}$ meson has been used to cut against random combinatoric background due to short lived particles. While CDF uses a minimum proper lifetime $c \tau$ of the $B_{s}^{0}$ candidate, $\mathrm{D} \varnothing$ is using decay length significance $L_{X Y} / \delta L_{X Y}$. The fragmentation characteristics of the $b$ quark are such that most of its momentum is carried by the $b$ hadron. The number of extra tracks near the $\mathrm{B}$ candidate therefore tends to be small. The second discriminant was therefore an isolation variable, $\mathcal{I}$, of the muon pair, defined as:

$$
\mathcal{I}=\frac{\left|\vec{p}\left(\mu^{+} \mu^{-}\right)\right|}{\left|\vec{p}\left(\mu^{+} \mu^{-}\right)\right|+\sum_{\operatorname{track} i \neq B} p_{i}(\Delta \mathcal{R}<1)}
$$


Here, $\sum_{\operatorname{track} i \neq B} p_{i}$ is the scalar sum over all tracks excluding the muon pair within a cone of $\Delta \mathcal{R}<1$ around the momentum vector $\vec{p}\left(\mu^{+} \mu^{-}\right)$of the muon pair where $\Delta \mathcal{R}=\sqrt{(\Delta \phi)^{2}+(\Delta \eta)^{2}}$. The CDF definition differs by using the transverse momentum of the tracks instead of the momentum.

As a third variable the angle $(\Delta \Phi)$ between the momentum vector of the muon pair and the vector from the primary to the secondary vertex has been used. This requirement ensures consistency between the direction of the decay vertex and the momentum vector of the $B_{s}^{0}$ candidate.

\section{Limit setting procedure and optimization}

CDF

For a given number of observed events, $n$, consistent with the background estimate, $n_{b g}$, the upper limit on the branching ratio is determined using:

$$
\mathcal{B}\left(B_{s} \rightarrow \mu^{+} \mu^{-}\right) \leq \frac{N\left(n, n_{b g}\right)}{2 \cdot \sigma_{B_{s}} \cdot \alpha \cdot \varepsilon_{\text {total }} \cdot \int \mathcal{L} d t}
$$

where $\mathrm{N}\left(n, n_{b g}\right)$ is the number of candidate decays at $90 \%$ C.L., estimated using a Bayesian approach 14 and incorporating the uncertainties into the limit. The integrated luminosity is $\mathcal{L}$, The $B_{s}^{0}$ production cross section is $\sigma_{B_{s}^{0}}\left(=\frac{f_{s}}{f_{u}} \sigma_{B^{+}}[15]\right.$ with $f_{s}=0.100, f_{u}=0.3912$ ) and $\alpha \cdot \varepsilon_{\text {total }}$ is the total acceptance times efficiency, obtained from data and Monte Carlo. The factor of two is necessary, since the analysis is sensitive to the charge conjugate $b$ hadron.

The optimization was done with approximately 100 combinations of the discriminating variables and maximized the a priori expected limit which is given by the sum over all possible observations, $n$, weighted by the corresponding Possion probability of the expected $n_{b g}$. The optimization of the discriminating variables $(\mathrm{c} \tau>200 \mu \mathrm{m}, \Delta \Phi<0.10 \mathrm{rad}, \mathcal{I}>0.65)$ predicted $1.05 \pm 0.30$ background events in $171 \mathrm{pb}^{-1}$.

$\mathrm{D} \emptyset$

A random grid search and an optimization procedure 13 was used to find the optimal cut values of the discriminating variables by maximizing the variable $P=\epsilon_{\mu \mu}^{B_{s}^{0}} /\left(a / 2+\sqrt{N_{\mathrm{bg}}}\right)$. Here, $\epsilon_{\mu \mu}^{B_{s}^{0}}$ is the reconstruction efficiency of the signal events relative to the preselection (estimated using MC), and $N_{\mathrm{bg}}$ is the expected number of background events interpolated from the sidebands. The constant $a$ is the number of standard deviations corresponding to the confidence level at which the signal hypothesis is tested. This constant $a$ was set to 2.0, corresponding to about the $95 \%$ C.L. The result $\left(L_{X Y} / \delta L_{X Y}>18.5, \Delta \Phi<0.2 \mathrm{rad}, \mathcal{I}>0.56\right)$ of the optimization leads to a background prediction of $3.7 \pm 1.1$ events in $240 \mathrm{pb}^{-1}$.

In the absence of an apparent signal a limit on the branching fraction $\mathcal{B}\left(B_{s}\right)$ can then be computed by normalizing the upper limit of number of events in the 
$B_{s}$ signal region to the number of reconstructed $B^{ \pm} \rightarrow J / \psi K^{ \pm}$events:

$$
\mathcal{B}\left(B_{s}\right) \leq \frac{N_{\mathrm{ul}}}{N_{B^{ \pm}}} \cdot \frac{\epsilon_{\mu \mu K}^{B^{ \pm}}}{\epsilon_{\mu \mu}^{B_{s}}} \cdot \frac{f_{b \rightarrow B_{s}}}{f_{b \rightarrow B_{u, d}}} \mathcal{B}_{1}\left(B^{ \pm}\right) \cdot \mathcal{B}_{2}(J / \psi),
$$

where $N_{\mathrm{ul}}$ is the upper limit on the number of signal decays estimated from the number of observed events and expected background events including systematic uncertainties using the Feldman and Cousins ordering scheme for the MC integration 16. The number of observed $B^{ \pm} \rightarrow J / \psi K^{ \pm}$events is $N_{B^{ \pm}}=$ $741 \pm 31 \pm 22, \epsilon_{\mu \mu}^{B_{s}} / \epsilon_{\mu \mu K}^{B^{ \pm}}=0.247 \pm 0.009 \pm 0.017$ is the efficiency ratio of the signal and normalization channels, obtained from Monte Carlo (MC) simulations. The fragmentation ratio of a $b$ or $\bar{b}$ quark producing a $B_{s}^{0}$ and a $B^{ \pm}$or $B_{d}$ is $f_{b \rightarrow B_{s}} / f_{b \rightarrow B_{u, d}}=0.270 \pm 0.034 \stackrel{12}{12}$ and $\mathcal{B}_{1}=\mathcal{B}\left(B^{ \pm} \rightarrow J / \psi K^{ \pm}\right)=(1.00 \pm 0.04) \cdot 10^{-3}$ and $\mathcal{B}_{2}=\mathcal{B}(J / \psi \rightarrow \mu \mu)=(5.88 \pm 0.1) \%$ are the measured branching ratios of the normalization channel.

\section{Results}

Both experiments have used a blind analysis technique, hiding the signal region until the analysis was completed. The invariant mass spectra after unblinding are shown in Fig. 1 No excess of a signal has been seen in either of the two experiments.
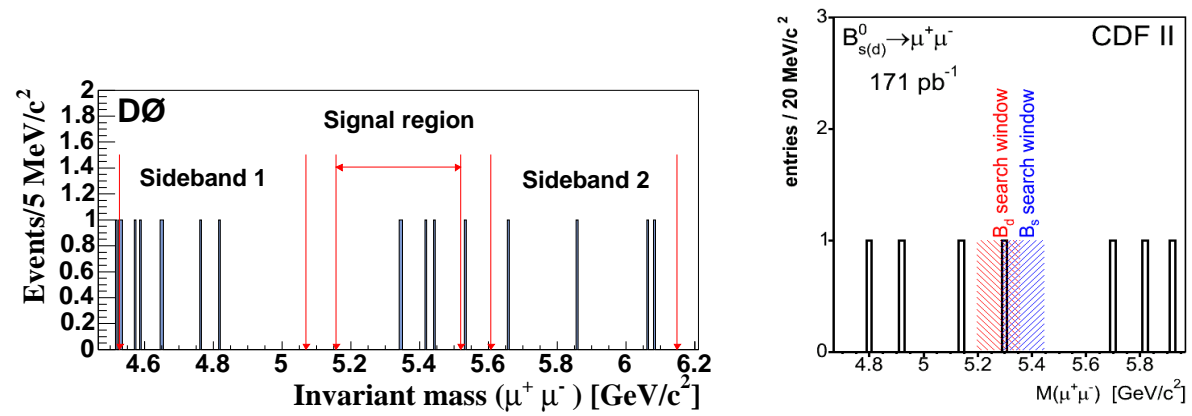

Fig. 1. The invariant mass spectrum for the D $\varnothing$ experiment (left side) and the CDF experiment (right side) after optimized requirements on the discriminating variables.

CDF observed one event with an invariant mass of $M_{\mu^{+} \mu^{-}}=5.295 \mathrm{GeV} / c^{2}$, thus falling into both the $B_{s}^{0}$ and $B_{d}^{0}$ mass window. Since this is consistent with the expected background of $1.1 \pm 0.3$ events in each of the mass windows $( \pm 80 \mathrm{MeV}$ around the corresponding PDG mass), 95\%(90\%) CL limits 17 of $\mathcal{B}\left(B_{s}^{0} \rightarrow \mu^{+} \mu^{-}\right)<$ $7.5 \cdot 10^{-7}\left(5.8 \cdot 10^{-7}\right)$ and $\mathcal{B}\left(B_{d}^{0} \rightarrow \mu^{+} \mu^{-}\right)<1.9 \cdot 10^{-7}\left(1.5 \cdot 10^{-7}\right)$ are found.

$\mathrm{D} \varnothing$ found four candidate events in the invariant mass region of $\pm 180 \mathrm{MeV}$ of the expected ${ }^{18} B_{s}^{0}$ mass, which is also consistent with the expectation of $3.7 \pm 1.1$ background events. This gives a resulting limit $\frac{19}{\text { on the branching fraction at } 95 \%(90 \%)}$ 
CL of $\mathcal{B}\left(B_{s}^{0} \rightarrow \mu^{+} \mu^{-}\right)<5.0 \cdot 10^{-7}\left(4.1 \cdot 10^{-7}\right)$. This limit assumes that there are no contributions from $B_{d}^{0} \rightarrow \mu^{+} \mu^{-}$decays in the search region. Any non-negligible contribution due to $B_{d}^{0}$ decays would make the obtained limit on the branching fraction smaller. The limit presented for $\mathcal{B}\left(B_{s}^{0} \rightarrow \mu^{+} \mu^{-}\right)$is therefore conservative and is even more stringent to constrain models of new physics beyond the SM.

\section{References}

1. Charge conjugated states are included implicitly.

2. G. Buchalla and A. J. Buras, Nucl. Phys B 400, 225 (1993); M. Misiak and J. Urban, Phys. Lett. B 451, 161 (1999); G. Buchalla and A. J. Buras, Nucl. Phys. B 548, 309 (1999).

3. A. J. Buras, Phys. Lett. B 566, 115 (2003).

4. F. Abe et al. [CDF Collaboration], Phys. Rev. D57(1998)3811.

5. H.E. Logan and U. Nierste, Nucl. Phys. B 586, 39 (2000).

6. S. R. Choudhury and N. Gaur, Phys. Lett. B 451, 86 (1999)

7. K. S. Babu and C. F. Kolda, Phys. Rev. Lett. 84, 228 (2000); A. Dedes et al., FERMILAB-PUB-02-129-T.

8. A. Dedes et al., Phys. Rev. Lett. 87, 251804 (2001).

9. T. Blazek et al., Phys. Lett B 589, 39 (2004); R. Dermisek et al., JHEP 0304, 37 (2003).

10. C. Bobeth et al., Phys. Rev. D 66, 074021 (2002).

11. R. Arnowitt et al. Phys. Lett. B 538, 121 (2002).

12. S. Eidelman et al., Phys. Lett. B 592, 1 (2004).

13. G. Punzi in Proc. of the Conference on Statistical Problems in Particle Physics, Astrophysics and Cosmology (Phystat 2003), edited by L. Lyons et al. (SLAC, Menlo Park, CA, 2003), p. 79 .

14. K. Hagiwara et al., Phys. Rev. D66, 010001 (2002).

15. D. Acosta et al. [CDF Collaboration], Phys. Rev. D65 052005(2002).

16. J. Conrad et al. Phys. Rev. D67, 012002 (2003).

17. D. Acosta et al. [CDF Collaboration], Phys. Rev. Lett.93 032001 (2004).

18. To compensate for a shift in the momentum scale of the $\mathrm{D} \varnothing$ tracking system, the expected $B_{s}^{0}$ mass is shifted downwards by $30 \mathrm{MeV} / c^{2}$ with respect to the PDG mass.

19. Abazov, V. M. et al. [D $\varnothing$ Collaboration], hep-ex/0410039 submitted to PRL. 